\title{
Análisis psicométrico del test de dependencia de videojuegos (TDV) en población peruana
}

\section{Psychometric analysis of the Test of Dependence of Videogames} (TDV) in Peruvian population

\author{
EDwin Salas-Blas ${ }^{\mathrm{a}}$ \\ Universidad de San Martín de Porres, Perú \\ ORCID: http://orcid.org/0000-0002-0625-0313 \\ César Merino-Soto \\ Universidad de San Martín de Porres, Perú \\ Mariano Chóliz \\ Universidad de Valencia, España \\ Clara Marco \\ Universidad de Valencia, España
}

a Autor de correspondencia. Correo electrónico:
e.salasb@hotmail.com

Para citar este artículo: Salas-Blas, E., Merino-Soto, C., Chóliz, M., \& Marco, C. (2017). Análisis psicométrico del test de dependencia de videojuegos (TDV) en población peruana. Universitas Psychologica, 16(4), 1-13. https://doi.org/10.11144/Javeriana.upsy16-4.apt d

\section{RESUMEN}

El objetivo del presente trabajo es validar en población peruana, el Test de Dependencia de Videojuegos (TDV) (Chóliz \& Marco, 2011). Los resultados obtenidos demuestran que se trata de una herramienta confiable y válida. Se encontró una sola estructura factorial, en lugar de cuatro dimensiones, como plantearon los autores del TDV. Este factor podría denominarse genéricamente: adicción a videojuegos, y podría servir como evidencia de la existencia de este trastorno, que coincidiría con el denominado 'Trastorno de Juego por Internet', que aparece en la Sección III del DSM-5. Los resultados hallados se discuten en relación a lo planteado por Chóliz y Marco (2011), en lo que se refiere a las diferencias en cuanto a sexo, edad y la estructura interna del instrumento.

\section{Palabras clave}

adicción a videojuegos; test de dependencia de videojuegos; adicciones tecnológicas; validación de cuestionario.

\footnotetext{
ABSTRACT

The objective of this work is the validation of the Test of Dependence of Videogames (TDV) (Chóliz \& Marco, 2011) in Peruvian population. The results show that it is a reliable and valid tool. We found a single factorial structure, rather than four dimensions as the authors of the TDV raised. This factor could be generically called: 'Addiction to video games', and could serve as evidence of the existence of this disorder, which would coincide with the so-called "Disorder online Game" listed in Section III of the DSM-5. The results found are discussed in relation to Chóliz \& Marco (2011) in terms of differences in sex, age and the internal structure of the instrument.

Keywords

videogame addiction; test of dependence of videogames; technological addictions; test validation.
} 


\section{Introducción}

Aun antes de los noventa del siglo pasado, ya existían algunos reportes que daban cuenta de las adicciones psicológicas, pero es a partir de Goldberg (1995), que este fenómeno se ha tratado con insistencia. Varias son las denominaciones que se utilizan para referirse a estos trastornos adictivos: adicciones comportamentales, sin droga, no químicas, sin sustancias, no tóxicas, etc. De este fenómeno general forman parte adicciones más específicas, entre ellas las adicciones a Internet, a las tecnologías, al móvil, a las compras, al sexo, a redes sociales, etc. La adicción a videojuegos (AVJ) que nos interesa en este trabajo, forma parte de este conjunto (Chóliz, 2006; Chóliz \& Marco, 2011; Escurra \& Salas 2014; Cruzado, Matos, \& Kendall, 2006; Echeburúa, 1999; Fernández-Montalvo \& Echeburúa, 1998; Griffiths, 2005; Luengo, 2004; Salas, 2014; Young, 1996a, 1996b).

En la revisión hecha acerca de AVJ, se han encontrado numerosos y diversos trabajos; un primer grupo revisa las bases teóricas para entenderlo como un trastorno, fundamenta por qué este tipo de comportamiento es una verdadera adicción, hace comparaciones con las adicciones a sustancias, generalmente partiendo de los criterios de la cuarta versión del Manual diagnóstico y estadístico de los trastornos mentales (DSM -IV), así como discute la atención que se le está dando en los últimos años al considerarlo como un problema de salud (Carbonell, Talarn, Beranuy, Oberst, \& Graner, 2009; Chóliz, 2006; Echeburúa, 1993; García, Montes, \& Alcol, 2012; Griffiths, 2005; Salas, 2014; Tejeiro, 2001; Tejeiro, Pelegrina, \& Gómez, 2009). Otro grupo de trabajos desarrolla descripciones de casos, de grupos relativamente pequeños, de modalidades y situaciones de juego específicas, con la finalidad de entender el problema a partir de perspectivas sociales, económicas y de salud (Arnao, Falla, \& Jiménez, 2011; Chóliz \& Mazón, 2011; Griffiths, 1991a, Griffiths \& Hunt, 1998). En un tercer grupo, se encuentran investigaciones que aportan técnicas e instrumentos que han sido creados para abordar o medir la AVJ, generalmente partiendo de los indicadores del DSM-IV para la dependencia de sustancias o para el juego patológico (Arnao et al., 2011; Chóliz \& Marco, 2011; Egli \& Meyers, 1984; Fisher, 1995; Griffiths 1991b). Estos instrumentos se fundamentan en técnicas de autorregistro, que tienen sus propias limitaciones debido a la subjetividad, a la deseabilidad social, las expectativas, etc., por lo que Tejeiro (2001), argumenta que se debe desconfiar de estos instrumentos y de los resultados hallados, porque son encuestas con poca sensibilidad y especificidad, y de las que podría dudarse su validez. No obstante, existen metodologías psicométricas que disponen de herramientas matemáticas que, precisamente, pretenden mejorar el proceso de evaluación de estos instrumentos, de manera tal que dicha advertencia debe tenerse más como un acicate para aumentar el rigor en la investigación, no para desechar uno de nuestros principales recursos de evaluación e investigación, como son los cuestionarios, autoinformes y autorregistros. Y este es uno de los principales objetivos del trabajo.

Entre las investigaciones revisadas, se han encontrado instrumentos cualitativos y cuantitativos. Algunos de estos instrumentos no se pueden evaluar psicométricamente porque descansan en fundamentos no cuantitativos como entrevistas o técnicas grupales y de análisis cualitativos (Arnao et al., 2011; Estallo, 1994); La mayoría de estos instrumentos no tienen referencias de confiabilidad, ni de validez analizadas desde varias fuentes de evidencias (como estructura interna, correlaciones con otras variables, etc.).

Una de las propuestas de medición más recientes de la AVJ es el Test de Dependencia de Videojuegos (TDV) construido y validado por Chóliz y Marco (2011), con población española. Fue construido sobre la base de los criterios para diagnosticar adicción a sustancias del DSM-IV. Este es uno de los pocos instrumentos en el área que cuenta con datos de la confiabilidad y validez que permite medir el fenómeno con objetividad y posibilita la réplica. 
Con datos obtenidos en Lima (Perú), en este trabajo se realiza un nuevo análisis psicométrico que permitirá evaluar la confiabilidad y la estructura interna del TDV, en un marco y contexto cultural diferente al español. Esto permitirá a los investigadores peruanos y potencialmente a los que se encuentran en el contexto latinoamericano, contar con un instrumento con evidencias de validez para estudiar la AVJ, que cada vez más, parece convertirse en un problema social, económico y de salud entre niños y adolescentes.

Chóliz y Marco (2011) describen los aspectos de la construcción del TDV, reportan su alta confiabilidad y en cuanto a la estructura interna, señalan en un primer momento que

... cada uno de los ítems poseen una correlación igual o superior a 0.5 con el resto de la escala, lo cual indica que todos los ítems miden el mismo constructo, en este caso, dependencia de videojuegos (y que) las dimensiones de las que consta el constructo (dependencia de videojuegos) no son independientes entre sí." (p. 422).

Sin embargo, más adelante describen el análisis factorial realizado, con el que hallaron cuatro factores, y muestran algunos aspectos metodológicos que podrían limitar la generalización de sus resultados. Específicamente, aplicaron un procedimiento usualmente desarrollado para reducir el número de variables (análisis de los componentes principales), que permite factorizar la varianza de todos los ítems, y también explicar la dependencia de los ítems en las dimensiones identificadas. Este método usa toda la varianza de los ítems, que incluye la varianza específica y otra común. Si bien esto permite usar la máxima variación posible, la varianza específica también contiene aquella proveniente del error (Nunnally \& Bernstein, 1995). En contraste con el análisis de componentes principales, el modelamiento de variables latentes en la construcción de pruebas psicológicas usa la varianza común, y es más recomendable para identificar los parámetros del constructo con menos sesgo (Widaman, 1993, 2007). Ambos enfoques son conceptualmente diferentes y pueden producir discrepancias en la estimación exacta y la estabilidad de los parámetros (De Winter \& Dodou, 2016).

Para la mejor interpretación de la solución componencial obtenida, Chóliz y Marco (2011) aplicaron una rotación promax (rotación oblicua), que asume que los factores están correlacionados; sin embargo, en su reporte no se encuentra la magnitud de las correlaciones entre los factores y/o entre los puntajes, por lo que se desconoce esta parte de la estructura interna del TDV. Luego, una vez obtenida la estructura factorial (en varios análisis realizados), usaron el puntaje total para describir y hacer correlaciones con otras variables; sin embargo, en los resultados reportados no se encontraron datos empíricos ni la racionalidad para crear un puntaje total. Sin contar con información de la covariación interfactorial, no se puede definir y validar las relaciones teóricas del constructo evaluado

A partir de la exploración inicial del trabajo de Chóliz y Marco (2011) se desprende que el TDV constituye un importante avance en la medición de la tendencia adictiva a los videojuegos. Esta es una escala Likert, que puede proporcionar información cuantitativa como puntaje total y por factores. Puede ser útil para propósitos descriptivos y, a partir de los mismos, generar planes de prevención tanto de grupos, como de individuos. También puede servir para realizar diagnósticos de la conducta adictiva, y para elaborar a partir de los resultados planes de intervención (por ejemplo, Chóliz, 2011; Marco \& Chóliz, 2014). Por las implicaciones de su uso, se requiere la replicación de sus propiedades métricas en otros contextos culturales como el peruano, que puede asegurar la interpretación generalizable del constructo latente.

Así pues, el objetivo principal de este trabajo es la validación en población peruana del TDV, para utilizarla en la investigación, en la evaluación y diagnóstico de la adicción a videojuegos, así como en el desarrollo de programas preventivos. Hay que enfatizar que en el Perú no existen herramientas similares, pese a que el problema de dependencia de los videojuegos se torna en un fenómeno social cada vez más importante. 


\section{Método}

\section{Participantes}

En el estudio participaron 467 escolares de educación básica regular, cuyas edades se encontraban en un rango de 11 a 18 años de edad $(11$ años $=0.2 \%, 12=5.14 \%, 13=8.35 \%, 14=$ $4.93 \%, 15=21.41 \%, 16=46.04 \%, 17=9.42 \%$ y $18=2.14 \%)$, de los cuales $266(56.96 \%)$ son varones. Cursaban entre el primer y quinto grado de estudios secundarios (1er grado $=21.6 \%, 2 \mathrm{do}$ grado $=35.1 \%$, 3er grado $=15.3 \%$, 4 to grado $=16.2 \%$ y 5 to grado $=11.7 \%$ ). Provienen de cinco instituciones educativas de gestión estatal regular, ubicadas en diferentes distritos de Lima Metropolitana, que pueden ser caracterizados como de nivel socioeconómico medio o bajo: Villa María del Triunfo $=29.1 \%$, Ate-Vitarte $=$ $14.1 \%$, Los Olivos $=16.7 \%$, Surquillo $=16.3 \%$, y Callao $=23.8 \%$, que fue la última institución en aceptar su participación, y fue seleccionada para evaluar la replicabilidad del análisis principal, es decir la validez de la estructura interna. Las instituciones fueron seleccionadas debido a la posibilidad de acceder a ellas. Los grados muestreados fueron elegidos aleatoriamente en cada institución, y dentro de ellos los estudiantes que conforman la muestra son aquellos que aceptaron voluntariamente ser parte del estudio respondiendo el cuestionario.

\section{Instrumento}

Test de Dependencia de Videojuegos (TDV) (Chóliz \&̊ Marco, 2011)

Se trata de un cuestionario de autorregistro que utiliza una escala ordinal, califica cero (0) por responder "totalmente en desacuerdo", uno (1) por "Un poco en desacuerdo", dos (2) por una respuesta "neutral", tres (3) por "un poco de acuerdo" y cuatro (4) por "totalmente de acuerdo". Fue elaborado por Chóliz y Marco (2011) usando los criterios del DSM-IVTR (American Psychiatric Association, 2000), correspondientes a la adicción a sustancias. Inicialmente redactaron 55 ítems a partir de estos criterios, que fueron validados por jueces. Producto del mismo, quedaron 32 ítems que luego del análisis psicométrico realizado, se redujeron a 25 ítems que componen finalmente la escala TDV.

El puntaje total del TDV posee una elevada consistencia interna $(\alpha=0.94)$, su estructura factorial fue derivada de un análisis de componentes, mediante el cual se extrajeron 4 factores, el primero Abstinencia $(40.43 \%$ de la varianza) compuesto por los ítems 3, 4, 6, 7, 10, $11,13,14,21$ y 25 ; un segundo factor llamado Abuso y Tolerancia, (5.49\% de la varianza), compuesto por 5 ítems: 1, 5, 8, 9 y 12; un tercer factor denominado Problemas ocasionados por los videojuegos, conformado por los ítems $16,17,19$ y 23 (4.19\% de la varianza); y el cuarto, llamado Dificultad en el control, (4\% de la varianza) constituido por los ítems 2, 15,18, 20, 22 y 24 .

En el presente estudio, se examinó lingüísticamente el instrumento para evaluar si era comprensible para la muestra, en este proceso participaron tres jueces/expertos (psicólogos/ docentes universitarios) quienes informaron que el instrumento en su versión original era adecuado para el presente estudio. Se realizó también una prueba piloto con 20 estudiantes del quinto año de secundaria de un colegio de gestión estatal, con los cuales se evaluó si existían ítems o palabras que presentaban dificultades para su comprensión. Se concluyó que no se requería de realizar modificaciones en el texto original, el mismo que fue aplicado a la muestra.

\section{Procedimiento}

Las autoridades, docentes y representantes de los padres de familia de las instituciones en las que se aplicó el cuestionario, aceptaron que este se administre, previa consulta a los propios estudiantes, a quienes se les explicó lo que se estaba desarrollando, evitando en todo momento hablar de dependencia o adicción por las implicancias de estos términos. Se 
acordó también guardar el anonimato de las instituciones y de los participantes.

Los cuestionarios fueron administrados en las aulas de clase en horario regular, dirigió el proceso por lo menos uno de los investigadores: el investigador entraba a las aulas y con el permiso del docente, solicitaba la colaboración voluntaria de los participantes. Se tuvo la colaboración de los de los docentes de aula y de los propios alumnos; los docentes y estudiantes participaron voluntariamente en el estudio. Se conformaron dos muestras, cuyos datos fueron tratados por separado para evaluar la replicabilidad de los resultados psicométricos.

La evaluación de la estructura interna se hizo mediante el modelamiento de ecuaciones estructurales (Bentler \& Chou, 1987; Byrne, 2006), para probar el modelo de medición del TDV. Primero, se hipotetizaron modelos que podrían representar la estructura latente del instrumento. El modelo unidimensional (M1) establece que la varianza de los ítems puede explicarse por un único y suficiente factor latente. El segundo modelo (M2) representa a los cuatro factores correlacionados hallados por Chóliz y Marco (2011). El último modelo (M3) es el bifactor, que establece que existe un factor general y cuatro factores específicos, que aportan cada uno varianza a los ítems (Chen, Hayes, Carver, Laurenceau, \& Zhang, 2012; Reise, 2012). Para propósitos de identificación de los modelos, el primer ítem de cada escala se fijó en 1. Los parámetros de los ítems se establecieron como se hace usualmente, donde la covariación entre el término de error de los ítems es igual a cero, y cada ítem carga solamente en un factor (Byrne, 2006). Luego de identificar el modelo más aceptable, se observaron los índices de modificación Lagrange (Sorbom, 1989) para las posibles re-especificaciones necesarias. Una vez que se verificaron las propiedades estructurales del TDV, se estimó la fiabilidad por consistencia interna, y finalmente se evaluaron las diferencias normativas.

Igualmente, se partió la muestra en dos grupos, con los que se realizó el mismo análisis estadístico, decisión que se tomó para confirmar los datos sobre la confiabilidad y la estructura interna del instrumento.

\section{Resultados}

Para la exposición de los resultados, primero se presentan de manera preliminar los datos descriptivos de los ítems; seguidamente, se muestran los resultados de la estructura interna y la fiabilidad por consistencia interna; finalmente, aparece la exploración de las diferencias normativas.

Respecto a los resultados descriptivos de los ítems (Tabla 1), se observa que las respuestas de los sujetos generalmente se ubican entre las tres primeras opciones (totalmente en desacuerdo, un poco en desacuerdo y neutral), señalando que la mayor densidad distribucional a nivel del ítem se ubica en el rechazo de las conductas de dependencia del TDV. Esto se confirma por la dirección de la asimetría, en la que todos los ítems muestran asimetría positiva. También se observa que la dispersión es mayor que los valores promedio, lo que sugiere que la cobertura de las respuestas se extiende a todas las opciones de los ítems.

\section{TABLA 1}

Estadísticos descriptivos y correlacionales para los items en la muestra total $(n=467)$

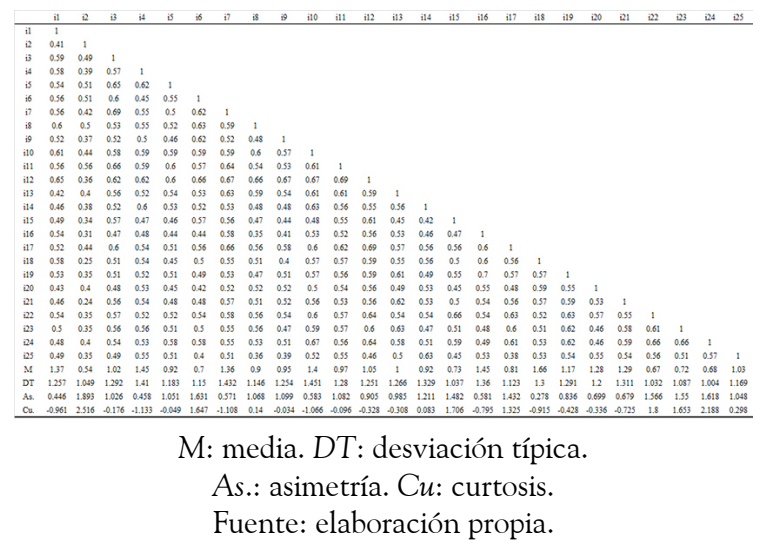

\section{Análisis de la estructura interna}

En el trabajo de Chóliz y Marco (2011), la presencia de un posible factor general se 
asumió debido a que se calculó un puntaje total, usado para las correlaciones de validez posteriores. Para verificar estos modelos, se aplicó un análisis factorial confirmatorio, mediante la función de estimación de máxima verosimilitud (Maximum Likelihood Estimate, MLE) sobre la matriz de correlaciones policóricas entre los ítems. Este es un procedimiento recomendado para analizar variables categóricas (Garrido, Abad, \& Ponsoda, 2013; Holgado-Tello, ChacónMoscoso, Barbero-García \& Vila-Abad, 2010). Para atenuar el impacto de la potencial falta de normalidad de los datos, se aplicó el ajuste Satorra y Bentler (1994) a la prueba de bondad de ajuste $\left(\mathrm{SB}-\chi^{2}\right)$. La evaluación del ajuste se complementó mediante varios indicadores de ajuste (Bentler \& Chou, 1987; Byrne, 2006; García-Cueto, Gallo, \& Miranda, 1998) para reducir la influencia sesgada de utilizar uno solo; específicamente, se usaron índices de absoluto (RMSEA < 0.05; SRMR < 0.08) y relativo $(\mathrm{CFI} \geq 0.95)$. Por último, se compararon la consistencia interna y la varianza extraída de cada modelo, mediante el procedimiento de Fornell y Larcker (1981), que compara la varianza común del factor con la varianza compartida entre las variables.

En general, se hallaron dos cosas: primero, existe una clara tendencia a encontrar valores de ajuste más satisfactorios en la muestra 2 comparados con la muestra 1, tal y como se describe en la Tabla 2, hecho que puede deberse a variaciones de las muestras, o a diferencias culturales. En segundo lugar, el ajuste de cada modelo es generalmente satisfactorio. El modelo más parsimonioso (M1, unidimensional) fue satisfactorio, similarmente al modelo oblicuo (M2), e incluso con un leve mejor ajuste en SRMR y CFI. Por otro lado, la correlación interfactorial estimada, desde este modelo (M2) se muestra en la Tabla 3, en donde se observa valores entre 0.8 y 0.97 . Este grado de correlación es muy alto comparado con el límite generalmente recomendado para identificar correlaciones elevadas ( $>0.5$; Cohen, 1988), lo que sugiere la existencia de fuerte dependencia entre constructos orientados a medir diferentes aspectos o factores de los mismos. Aunque estadísticamente este modelo produce menor discrepancia en el ajuste, es razonable considerarlo conceptualmente redundante debido al tamaño de las correlaciones. El modelo final, correspondiente al bifactor (M3), presentó el mejor ajuste; sin embargo, se hallaron problemas de convergencia debido a que se obtuvo una matriz definida no positiva. Tomando en cuenta la evaluación del M2, en el que se hallaron correlaciones interfactoriales muy altas, se sospechó que el problema sería la dependencia lineal entre las correlaciones de algunas variables.

\section{TABLA 2}

Resultados del ajuste de los modelos evaluados

\begin{tabular}{lllll}
\hline Modelos & SB- $\chi^{2}$ & RMSEA (IC 90\%) & SRMR CFI \\
\hline M1 (gl=275) & & & & \\
Muestra 1 & 524.386 & $0.051(0.044,0.057)$ & 0.048 & 0.992 \\
Muestra 2 & 329.914 & $0.043(0.021,0.059)$ & 0.076 & 0.988 \\
M2 (gl=269) & & & & \\
Muestra 1 & 479.789 & $0.047(0.04,0.054)$ & 0.047 & 0.993 \\
Muestra 2 & 31.213 & $0.037(0.008,0.055)$ & 0.077 & 0.991 \\
M3 (gl=250) & & & & \\
Muestra 1 & 385.408 & $0.039(0.031,0.046)$ & 0.044 & 0.996 \\
Muestra 2 & 265.154 & $0.023(0,0.046)$ & 0.07 & 0.996 \\
\hline
\end{tabular}

M1: modelo unidimensional. M2: modelo de factores oblicuos. M3: modelo bifactor. Fuente: elaboración propia. 
TABLA 3

Correlación inter-factorial estimada desde el modelo de factores oblicuos (M2)

\begin{tabular}{lcccc}
\hline & F1 & F2 & F3 & F4 \\
\hline Muestra 1 & & & & \\
F1 & 1 & & & \\
F2 & 0.961 & 1 & & \\
F3 & 0.936 & 0.896 & 1 & \\
F4 & 0.967 & 0.933 & 0.976 & 1 \\
Muestra 2 & & & & \\
F1 & 1 & & & \\
F2 & 0.899 & 1 & & \\
F3 & 0.882 & 0.813 & 1 & \\
F4 & 0.92 & 0.842 & 0.913 & 1 \\
\hline
\end{tabular}

Fuente: elaboración propia.

Para corregir este problema y obtener una estimación evaluable del modelo (M3), se reespecificaron algunos valores de inicio; para ello, se corrió nuevamente el análisis con el método de los mínimos cuadrados (Least Squares) para verificar la fuente del sesgo (Bentler \& Whu, 2004; Byrne, 2006). Efectivamente, en la muestra 1 se detectaron varianzas negativas en los ítems 1 , 3 y 16; y, en la muestra 2, esto mismo ocurrió en los ítems 19 y 22. Estas varianzas se fijaron en cero (Gerbing \& Anderson, 1987) y se corrió nuevamente el análisis, esta vez con el método ML, con el que se logró obtener las estimaciones que permitieron evaluar el modelo bifactor; los resultados de los parámetros individuales se muestran en la Tabla 4 para el modelo bifactor. En ambas muestras, las cargas de los factores específicos se acercan a cero, otros valores no razonables ocurrieron también, como cargas negativas (por ejemplo, ítem 25 y 14 en la muestra 1), y cargas con excesiva intensidad (ítem 19 y 22 en la muestra 1 ; ítem 25 y 18 en la muestra 2). Debido a la comunidad $=1$ de estas cargas, pueden reemplazarse por cero. La confiabilidad estimada por omega jerárquico $\left(\omega_{H}\right.$; Zinbarg, Yovel, Revelle, \& McDonald, 2006) informó que toda la varianza confiable proviene del factor general en ambas muestras, mientras que las magnitudes de $\omega_{H}$ en los factores individuales perdieron toda la varianza explicada.

TABLA 4

Parámetros individuales para el modelo bi-factor, en ambas muestras

\begin{tabular}{|c|c|c|c|c|c|c|c|c|c|c|c|c|c|}
\hline & \multicolumn{7}{|c|}{ Muestra $1(n=356)$} & \multicolumn{6}{|c|}{ Muestra 2( $n=111)$} \\
\hline & & F1 & $\mathrm{F} 2$ & $\mathrm{~F} 3$ & F4 & $\mathrm{Fg}$ & $\frac{h^{2}}{2}$ & $\mathrm{Fi}$ & $\mathrm{F} 2$ & $\mathrm{~F} 3$ & F4 & $\mathrm{Fg}$ & $h^{2}$ \\
\hline Item 3 & & 0.11 & & & & 0.99 & 1 & 0 & & & & 0.75 & 0.56 \\
\hline Item 4 & & -0.22 & & & & 0.73 & 0.58 & 0 & & & & 0.48 & 0.24 \\
\hline İtem 6 & & 0.19 & & & & 0.75 & 0.59 & 0 & & & & 0.59 & 0.34 \\
\hline Item 7 & & 0.09 & & & & 0.79 & 0.64 & 0 & & & & 0.7 & 0.49 \\
\hline Item 10 & & -0.09 & & & & 0.79 & 0.63 & 0 & & & & 0.67 & 0.45 \\
\hline Item 11 & & -0.01 & & & & 0.8 & 0.63 & 0 & & & & 0.7 & 0.5 \\
\hline Item 13 & & -0.05 & & & & 0.75 & 0.57 & 0 & & & & 0.73 & 0.54 \\
\hline Item 14 & & -0.35 & & & & 0.71 & 0.62 & -0.01 & & & & 0.71 & 0.5 \\
\hline İtem 21 & & -0.13 & & & & 0.72 & 0.54 & 0 & & & & 0.47 & 0.22 \\
\hline Item 25 & & -0.41 & & & & 0.66 & 0.6 & 0 & & & & 0.62 & 1 \\
\hline Ítem 1 & & & 0.18 & & & 0.98 & 1 & & 0.47 & & & 0.58 & 0.56 \\
\hline Item 5 & & & 0.01 & & & 0.72 & 0.52 & & -0.14 & & & 0.68 & 0.49 \\
\hline Item 8 & & & 0.13 & & & 0.73 & 0.54 & & 0.37 & & & 0.59 & 0.48 \\
\hline Item 9 & & & 0.22 & & & 0.68 & 0.51 & & 0.1 & & & 0.6 & 0.37 \\
\hline Item 12 & & & 0.49 & & & 0.83 & 0.93 & & 0.54 & & & 0.69 & 0.76 \\
\hline Item 16 & & & & -0.01 & & 1 & 1 & & & 0.48 & & 0.71 & 0.74 \\
\hline Item 17 & & & & 0 & & 0.78 & 0.61 & & & 0.15 & & 0.63 & 0.42 \\
\hline Item 19 & & & & 0 & & 0.73 & 1 & & & 0.43 & & 0.9 & 1 \\
\hline Item 23 & & & & 0 & & 0.74 & 0.54 & & & 0.12 & & 0.65 & 0.44 \\
\hline İtem 2 & & & & & 0 & 1 & 1 & & & & 0 & 0.56 & 0.32 \\
\hline Item 15 & & & & & 0 & 0.69 & 0.47 & & & & 0 & 0.55 & 0.31 \\
\hline Item 18 & & & & & 0 & 0.72 & 0.52 & & & & 0 & 0.61 & 1 \\
\hline Item 20 & & & & & 0 & 0.67 & 0.45 & & & & 0 & 0.48 & 0.23 \\
\hline Item 22 & & & & & 0 & 0.76 & 1 & & & & 0 & 1 & 1 \\
\hline Item 24 & & & & & 0 & 0.76 & 0.58 & & & & 0 & 0.72 & 0.52 \\
\hline & $\omega_{H}$ & 0 & 0 & 0 & 0 & 0.97 & & 0 & 0 & 0 & 0 & 0.94 & \\
\hline
\end{tabular}

Fi: variables latentes específicas. Fg: variable latente general. $h^{2}$ : confiabilidad del ítem. $\omega_{H}$ : omega jerárquico. Fuente: elaboración propia.

La evaluación sugiere que el modelo unidimensional puede describir parsimoniosamente y de manera efectiva, las relaciones entre los ítems en ambas muestras. Los parámetros individuales de este modelo aparecen en la Tabla 5. Todas las cargas son estadísticamente significativas $(z>4)$, y pueden considerarse elevadas ( $>0.5$; McDonald, 1999; Nunnally \& Bernstein, 1995). 


\section{TABLA 5}

Cargas factoriales en el modelo unidimensional

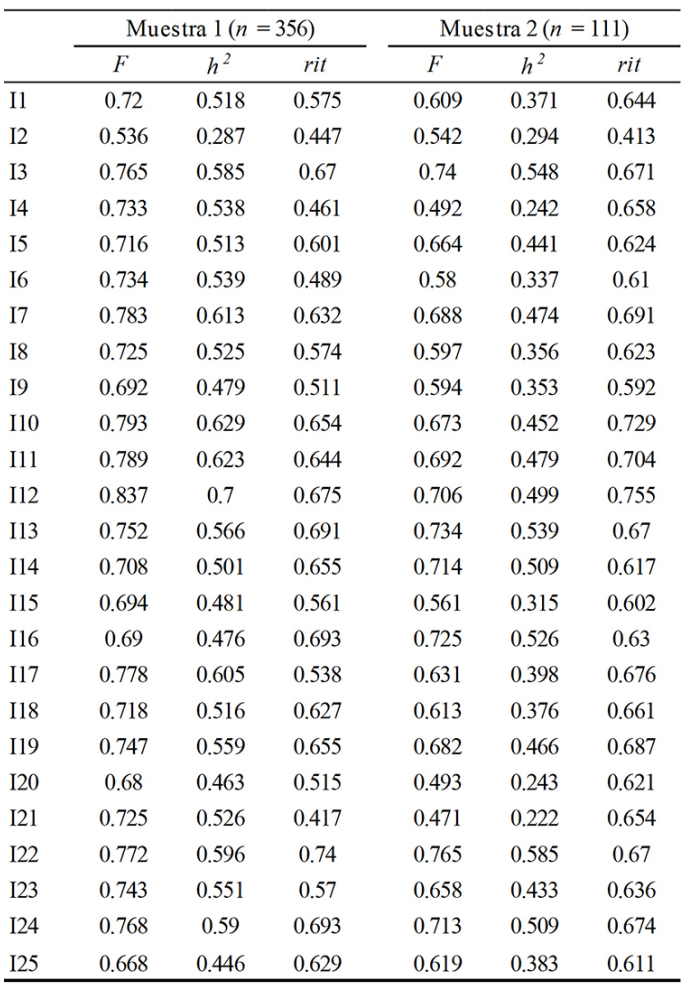

F: cargas del factor. $h^{2}$ : comunalidad. $r_{i t}$ : correlación ítem-test corregida.

Fuente: elaboración propia.

\section{Confiabilidad}

La confiabilidad por consistencia interna se estimó por el coeficiente a (Cronbach, 1951; Yanyun \& Green, 2011) y sus intervalos de confianza (Romano, Kromrey, Owens, \& Scott, 2011; Romano, Kromrey, \& Hibbard, 2012), mediante el de Feldt. Desde el método factorial, se obtuvo el coeficiente $w$ (McDonald, 1999). Los coeficientes $\alpha$ hallados en la muestra 1 (0.951, IC 95\%: 0.943 - 0.958) y la muestra 2 (0.938, IC 95\%: 0.921 - 0.954), indican que ambos son indistinguibles respecto a su magnitud. La confiabilidad $w$ estimada por el coeficiente para la muestra $1(0.96)$ y muestra 2 (0.94), señala elevada varianza confiable, ya que son altamente similares a lo estimado con el coeficiente $\alpha$. Esta similaridad puede estar relacionada con la equivalencia de las magnitudes de las cargas factoriales (presentadas en la Tabla 5), así como la presencia de triviales errores correlacionados entre los ítems. Dada la sencillez de su cálculo, se sugiere que el coeficiente $\alpha$ es el estimador aceptable para esta muestra. Por otro lado, la correlación interítem $\left(r_{i i}\right)$ en ambas muestras (0.43 y 0.38 , respectivamente) está comprendida entre los límites sugeridos para instrumentos homogéneos (Clark \& Watson, 1995).

\section{Diferencias normativas}

En la regresión lineal, el efecto del sexo y edad sobre el puntaje total fue estadísticamente significativo, $F(2.464)=18.066, p<0.001$. El modelo explicó el $6.8 \%$ ( $\mathrm{R}$ a j $2=0.068, \mathrm{R}^{2}=$ 0.072 ) de la variabilidad del puntaje total. Los resultados específicos indican que el sexo tuvo más impacto sobre el puntaje total $(\mathrm{B}=11.41$, error estándar $=1.908, \mathrm{~b}=0.271, t=5.978$, $p<0.001 ;$ constante $=47.25$, error estándar $=$ 11.58). El impacto de la edad puede considerarse dentro del error de muestreo, $(B=-0.264$, error estándar $=0.701, \mathrm{~b}=-0.017, \mathrm{t}=-0.377, \mathrm{p}>$ $0.05)$. El sexo en la muestra total produce una diferencia estandarizada igual a $\mathrm{g}=0.566$, una magnitud moderadamente fuerte (Cohen, 1988), y demuestra que los hombres obtienen mayor puntaje que las mujeres en cada nivel de edad.

Se examinó la variabilidad del puntaje respecto al sexo e institución educativa ( 5 niveles de grado), mediante un ANOVA factorial $2 \times 5$. El efecto total de estas variables sobre el puntaje fue $R^{2}=0.154(\mathrm{R}$ a j $2=0.137)$. La interacción sexo e institución apenas se acercó al nivel de significancia, $F(4.547)=2.362, p=$ $0.052, \eta_{\text {parcial }}^{2}=0.02$; pero los efectos principales provenientes de la institución $(F[4.457]=8.91$, $p<0.001)$ y sexo $(F[1.457]=27.23, p<$ $0.001)$ sí tuvieron influencia más allá del error de muestreo. Esto indica que la tendencia al videojuego varía fuertemente de acuerdo a la institución específica muestreada, y de manera similar ocurre con el sexo, hallazgo también descrito en el párrafo anterior. El grado de influencia más grande proviene de la institución 
$\left(\eta^{2}\right.$ parcial $\left.=0.072\right)$ comparado con la influencia del sexo $\left(\eta^{2}\right.$ parcial $\left.=0.056\right)$, pero este último puede considerarse también un efecto grande. En la Tabla 6 aparecen los puntajes promedio de las instituciones según el sexo. El grado de diferencia entre varones y mujeres va desde un nivel trivial (institución de Surquillo) hasta una diferencia sustancial entre los puntajes (institución de Villa María del Triunfo).

\section{TABLA 6}

Estadísticos descriptivos del puntaje total en cada instrucción participante

\begin{tabular}{|c|c|c|c|c|c|c|c|c|c|}
\hline \multirow[t]{2}{*}{ Institución } & \multicolumn{3}{|c|}{ Mujer } & \multicolumn{3}{|c|}{ Hombre } & \multicolumn{3}{|c|}{$\begin{array}{l}\text { Magnitud del efecto } \\
\text { (IC } 95 \% \text { ) }\end{array}$} \\
\hline & $\mathrm{M}$ & $\mathrm{DE}$ & $\mathrm{N}$ & $\mathrm{M}$ & $\overline{\mathrm{DE}}$ & $\mathrm{N}$ & $g$ & Inf. & Sup. \\
\hline $\begin{array}{l}\text { Villa María de } \\
\text { Triunfo }\end{array}$ & 10.64 & 16.59 & 64 & 29.79 & 20.85 & 72 & -1 & -0.64 & 1.35 \\
\hline Ate-Vitarte & 25.48 & 14.77 & 23 & 35.86 & 20.56 & 43 & -0.54 & -0.03 & 1.05 \\
\hline Los Olivos & 17.11 & 18.36 & 27 & 24.82 & 18.31 & 51 & -0.41 & -0.05 & 0.88 \\
\hline Surquillo & 32 & 20.37 & 29 & 35.4 & 20.38 & 47 & -0.16 & -0.29 & 0.62 \\
\hline Callao & 25.03 & 19.32 & 58 & 34.42 & 21.14 & 53 & -0.46 & 0.08 & 0.83 \\
\hline Total & 20.44 & 17.95 & 201 & 31.73 & 20.29 & 266 & -0.58 & -2.33 & 1.16 \\
\hline
\end{tabular}

G: $g$ de Hedges

Fuente: elaboración propia.

\section{Conclusiones y Discusión}

La adicción a videojuegos es uno de los principales problemas que ocasiona el uso desadaptativo de las denominadas nuevas tecnologías. A pesar de que la única adicción comportamental categorizada como tal en el DSM-5 (American Psychiatric Association, 2013) es la adicción al juego de azar, este mismo manual reconoce que han recogido 240 artículos sobre el tema, y se ha encontrado evidencia de la similitud entre el juego online y el resto de adicciones, tanto al juego de azar como a las drogas. Igualmente, la Asociación Americana de Psiquiatría considera el juego online (lo que podríamos denominar adicción a videojuegos online), como un problema clínico digno de ubicarse en la Sección III. Estimamos probable que en el momento en el que haya más evidencia científica de este problema, la APA considere la categorización de la adicción a videojuegos como la segunda adicción comportamental, ya que ya es tipificado como trastorno adictivo, y aparece como tal, en revistas médicas de Asia y EEUU.
Atendiendo a esta cuestión, el presente trabajo pretende validar el Test de Dependencia de Videojuegos (Chóliz \& Marco, 2011) en población peruana, con el objetivo de adaptar para esta población herramientas que ayuden a identificar el problema, que sean útiles en el diagnóstico y sirvan para el estudio y análisis del fenómeno y de los problemas que con él se relacionan.

Las diferencias encontradas al comparar los datos por género eran algo que se esperaba, ya que los varones de un contexto social con el que se ha trabajado, y en general de la sociedad peruana, tienen mayores oportunidades de involucrarse en juegos de esta naturaleza, en donde los padres asumen una actitud más contemplativa con ellos, cosa que no sucede con las mujeres, a quienes se les restringe este tipo de prácticas dada su "condición de mujer". A pesar de las eventuales diferencias culturales entre Perú y España, este dato es compatible con los hallados por Chóliz y Marco (2011) en población española, quienes encuentran las mismas diferencias y describen que los varones juegan más tiempo, con más frecuencia y más variedad de juegos que las mujeres; ellos prefieren deportes, aventuras y violencia, mientras que las mujeres prefieren juegos en los cuales se desarrollan aspectos afectivos. Estos datos son también congruentes con otros hallados en el campo de otras adicciones tecnológicas como es caso del móvil (Chóliz, Villanueva, \& Chóliz, 2009) o de las redes sociales (Salas \& Escurra, 2014).

Las diferencias encontradas por la edad fueron triviales y estadísticamente no significativas, lo cual es explicable por el hecho de que los videojuegos probablemente sean la herramienta tecnológica cuyo uso aparece de forma más temprana (ya que se comienza a jugar con dispositivos electrónicos en la infancia antes de que se tenga acceso a móviles o Internet) y se mantiene a lo largo del desarrollo (los videojuegos son actividades de las más preferidas en los momentos de ocio durante la niñez, adolescencia y juventud).

Respecto a la confiabilidad, se halló que las estimaciones producidas desde la teoría clásica de 
los tests y desde el modelamiento de ecuaciones estructurales fueron altamente similares, hecho que sugiere que la homogeneidad de los ítems puede satisfacer el modelo tau-equivalente, y calcular válidamente del coeficiente coeficiente $\alpha$ (Schweizer, 2011; Sijtsma, 2009). Aunque esto no fue corroborado directamente mediante el modelamiento estadístico, observando las cargas factoriales puede llegarse a esta conclusión. Esto es importante porque permite obtener una estimación de la confiabilidad sin sesgos en este parámetro, y usarlo para obtener puntajes verdaderos y criterios de diferencias confiables en la comparación inter-grupos e intra-individual.

Finalmente, otra cuestión relevante, la constituyen los resultados hallados acerca de la estructura factorial del cuestionario; en concreto, la consideración de uno o cuatro factores. El análisis realizado en el presente estudio, contempló tres modelos: el primero, que arroja la existencia de un solo factor debido a que todos los ítems están altamente correlacionados; el segundo modelo, que busca precisamente determinar la existencia de los cuatro factores reportados por Chóliz y Marco (2011) que se detectan en el análisis realizado, pero las altas correlaciones entre los ítems nos inducen a proponer la existencia de un factor principal y común en el que los ítems pueden unificarse (dependencia a videojuegos). El tercer modelo, el bifactor, resulta determinante para arbitrar y fundamentar el reconocimiento de la presencia de un único factor (dependencia a videojuegos) que agrupa a todos los ítems del TDV. Este mismo hallazgo fue reportado por Chóliz y Marco (2011) dentro de un análisis inicial, pero luego determinan la existencia de cuatro factores, debido a que el constructo de dependencia consiste en diferentes dimensiones que no son independientes entre sí. Así, por ejemplo, los autores argumentan que el abuso de videojuegos está relacionado con los problemas que ello acarrea, por ejemplo. Sin embargo, en el caso de la muestra peruana y por los análisis realizados, se sugiere la existencia de un solo factor, motivo por el cual se considera necesario seguir investigando, lo cual es una necesidad enfatizada en el propio DSM-5 (American Psychiatric Association, 2013).

Una posible limitación del estudio es el hecho de que se hayan obtenido diferencias entre los participantes de las instituciones, lo cual indica la existencia de eventuales diferencias sociológicas entre las muestras, cuestión que no pudo constatarse durante el procedimiento de muestreo. Esto, emperamos, plantea nuevos interrogantes, como es el de la relevancia de las variables sociológicas, sociodemográficas o económicas en el patrón de uso y eventual adicción a los videojuegos, hecho que por su relevancia, deberá ser investigado en ulteriores estudios científicos.

\section{Agradecimientos}

A la Facultad de Ciencias de la Comunicación, Turismo y Psicología de la Universidad de San Martín de Porres que asumió el costo de todo el proceso de la investigación.

\section{Referencias}

American Psychiatric Association. (2000). Diagnostic and statistical manual of mental disorders (4th ed., Text Revision). Washington, DC: American Psychiatric Association.

American Psychiatric Association. (2013). Diagnostic and statistical manual of mental disorders (5th ed.). Washington, DC: American Psychiatric Association.

Arnao, J., Falla, G., \& Jiménez, A. (2011). Los juegos en línea en adolescentes y jóvenes. Un estudio cuanti-cualitativo, descriptivo y analítico en jóvenes del Perú. Lima: Centro de Información y Educación para la Prevención del Abuso de Drogas (CEDRO).

Bentler, P. M., \& Chou, C. P. (1987). Practical issues in structural modeling. Sociological Methods EO Research, 16(1), 78-117. https:// doi.org/10.1177/0049124187016001004 
Bentler, R. M., \& Wu, E. J. C. (2004). EQS 6.1 for windows [Statistical Program]. Encino, CA: Multivariate Software, Inc.

Byrne, B. M. (2006). Structural equation modeling with EQS: Basic concepts, applications, and programming. Mahwah, NJ: Lawrence Erlbaum Associates

Carbonell, X. Talarn, A., Beranuy, M., Oberst, U., \& Graner, C. (2009). Cuando jugar se convierte en un problema: el juego patológico y la adicción a los juegos de rol online. Aloma, Revista de Psicología, 25, 201-220.

Chen, F. F., Hayes, A., Carver, C. S., Laurenceau, J.-P., \& Zhang, Z. (2012). Modeling general and specific variance in multifacted constructs: A comparison of the bifactor model to other approaches. Journal of Personality, 80(1), 219-251. https:// doi.org/10.1111/j.1467-6494.2011.00739.x

Chóliz, M. (2006). Adicción al juego de azar. Buenos Aires: Elaleph.

Chóliz, M. (2011). PrevTec 3.1: Programa de Prevención de Adicciones Tecnológicas. Valencia: FEPAD.

Chóliz, M., \& Mazón, M. (2011). El efecto de la crisis económica sobre el gasto en juego de azar. Información Psicológica, 101, 4-13.

Chóliz, M., \& Marco, C. (2011). Patrón de uso y dependencia de videojuegos en infancia y adolescencia. Anales de Psicología, 27(2), 418-426.

Chóliz, M., Villanueva, V., \& Chóliz, M. C. (2009). Ellas, ellos y su móvil: uso, abuso (iy dependencia?) del teléfono móvil en la adolescencia. Revista Española de Drogodependencias, 34(1), 74-88

Clark, L. A., \& Watson, D. (1995). Constructing validity: Basic issues in objective scale development. Psychological Assessment, 7(3), 309-319. https:// doi.org/10.1037//1040-3590.7.3.309

Cohen, J. (1988). Statistical power analysis for the behavioral sciences (2nd ed.). Hillsdale, NJ: Lawrence Earlbaum Associates.

Cronbach, L. J. (1951). Coefficient alpha and the internal structure of tests. Psychometrika,
16(3), 297-334. https://doi.org/10.1007/ BF02310555

Cruzado, L., Matos, L., \& Kendall, R. (2006). Adicción a internet: perfil clínico y epidemiológico de pacientes hospitalizados en un instituto nacional de salud mental. Revista Médica Herediana, 17(4), 196-205.

De Winter, J. C. F., \& Dodou, D. (2016). Common factor analysis versus principal component analysis: a comparison of loadings by means of simulations. Communications in Statistics - Simulation and Computation, 45(1), 299-321. https:// doi.org/10.1080/03610918.2013.862274

Echeburúa, E. (1999). ¿Adicciones sin drogas? Las nuevas adicciones: juego, sexo, comida, compras, trabajo, internet. Bilbao: Desclee de Brower.

Echeburúa, E. (1993). Las conductas adictivas: ¿Una ruta común desde el crack al juego patológico? Psicología Conductual, 1(3), 321-337.

Egli, E. A., \& Meyers, L. S. (1984). The role of videogame playing in adolescent life: Is there reason to be concerned? Bulletin of Psychonomic Society, 22 (4), 309-312. https:// doi.org/10.3758/bf03333828

Escurra, L.M., \& Salas, E. (2014). Construcción y validación del cuestionario de adicción a redes sociales (ARS). Liberabit, 20(1), 73-91

Estallo, J. A. (1994). Videojuegos, personalidad y conducta. Psicothema, 6(2), 181-190

Fernández-Montalvo, J., \& Echeburúa, E. (1998). Laborodependencia: Cuando el trabajo se convierte en adicción. Revista de Psicopatología y Psicología Clínica, 3(2), 103-120. https://doi.org/10.5944/ rppc.vol.3.num.2.1998.3860

Fisher, S. E. (1995). The amusement arcade as a social space for adolescents: an empirical study. Journal of Adolescence, 18(1), 71-86. https://doi.org/10.1006/jado.1995.1006

Fornell, C., \& Larcker, D. F. (1981). Evaluating structural equation models with unobservable variables and measurement error. Journal of Marketing Research, 18(1), 39-50. https://doi.org/10.2307/3151312 
García, A., Montes, C., \& Alcol, I. (2012). El juego patológico en los adolescentes. Psicología de las adicciones, 1, 29-34.

García-Cueto, E., Gallo, P., \& Miranda, R. (1998). Bondad de ajuste en el análisis factorial confirmatorio. Psicothema, 10(3), 717-723.

Garrido, L. E., Abad, J. J., \& Ponsoda, V. (2013). A new look at Horn's parallel analysis with ordinal variables. Psychological Methods, 18(4), 454-474. https://doi.org/10.1037/ a0030005

Gerbing, D. W., \& Anderson, J. C. (1987). Improper solutions in the analysis of covariance structures: Their interpretability and a comparison of alternate respecifications. Psychometrika, 52(1), 99-111. https://doi.org/10.1007/ BF02293958

Goldberg, I. (1995). Internet Addiction disorder. Diagnostic criteria. Recuperado el 1-09-2014 de: http://www.iucf.indiana.edu /brown/hyplan/addict.html.

Griffiths, M. D. (1991a). The observational study of adolescent gambling in UK amusement arcades. Journal of Community and Applied Social Psychology, 1(4), 309-320. https:// doi.org/10.1002/casp.2450010406

Griffiths, M. D. (1991b). Amusement machine playing in childhood and adolescence: A comparative analysis of video games and fruit machines. Journal of Adolescence, 14(1), 53-73. https:// doi.org/10.1016/0140-1971(91)90045-S

Griffiths, M. D. (2005). Adicción a los videojuegos: una revisión de la literatura. Psicología conductual, 13, 445-462.

Griffiths, M. D., \& Hunt, N. (1998). Dependence on computer games by adolescents. Psychological Reports, 82(2), 475-480. https://doi.org/10.2466/PR0.82.2.475-480

Holgado-Tello, F.P., Chacón-Moscoso, S., Barbero-García, I., \& Vila-Abad, E. (2010). Polychoric versus Pearson correlations in exploratory and confirmatory factor analysis of ordinal variables. Quality and Quantity, 44, 153-166. https:// doi.org/10.1007/s11135-008-9190-y
Luengo, A. (2004). Adicción a internet: conceptualización y propuesta de intervención. Revista Profesional Española de Terapia Cognitivo-Conductual, 2, 22-52.

Marco, C., \& Chóliz, M. (2014). Tratamiento cognitivo-conductual de la adicción a videojuegos de rol online: Fundamentos de propuesta de tratamiento y estudio de caso. Anales de Psicología, 30(1), 46-55. https:// doi.org/10.6018/analesps.30.1.150851

McDonald, R. P. (1999). Test theory: A unified treatment. Mahwah, NJ: Erlbaum.

Nunnally, J. C., \& Bernstein, I. H. (1995). Teoría psicométrica. México, DF: McGraw-Hill.

Reise, S. P. (2012). The rediscovery of bifactor measurement models. Multivariate Behavioral Research, 47(5), 667-696. https:// doi.org/10.1080/00273171.2012.715555

Romano, J. L., Kromrey, J. D., \& Hibbard, S. T. (2012). A Monte Carlo study of eight confidence interval methods for coefficient alpha. Educational and Psychological Measurement, 70(3), 376-393. https:// doi.org/10.1177/0013164409355690

Romano, J. L., Kromrey, J. D., Owens, C. M., \& Scott, H. M. (2011). Confidence interval methods for coefficient alpha on the basis of discrete, ordinal response items: Which one, if any, is the best? The Journal of Experimental Education, 79(4), 382-403. https:// doi.org/10.1080/00220973.2010.510859

Salas, E. (2014). Adicciones psicológicas y los nuevos problemas de salud. Cultura, 28, $111-146$

Salas, E., \& Escurra, M. (2014). Uso de redes sociales entre estudiantes universitarios limeños. Revista Peruana de Psicología y Trabajo Social, 3, 75-90

Satorra, A., \& Bentler, P. M. (1994). Corrections to test statistics and standard errors in covariance structure analysis. In A. von Eye \& C. C. Clogg (Eds.), Latent variables analysis: Applications for developmental research (pp. 399-419). Thousand Oaks, CA: Sage.

Schweizer, K. (2011). On the changing role of Cronbach's alpha in the 
evaluation of the quality of a measure. European Journal of Psychological Assessment, 27, 143-144. https:// doi.org/10.1027/1015-5759/a000069

Sijtsma, K. (2009). On the use, misuse, and the very limited usefulness of Cronbach's alpha. Psychometrika, 74, 107-120. https:// doi.org/10.1007/s11336-008-9101-0

Sorbom, D. (1989). Model modification. Psychometrika, 54(3), 371-384. https:// doi.org/10.1007/BF02294623

Tejeiro, R. (2001). La adicción a los videojuegos. Una revisión. Adicciones, 13(4), 407-413. https://doi.org/ 10.20882/adicciones.555

Tejeiro, R., Pelegrina, M., \& Gómez, J. L. (2009). Efectos psicosociales de los videojuegos. Comunicación, 1(7), 235-250.

Widaman, K. F. (1993). Common factor analysis versus principal component analysis: Differential bias in representing model parameters? Multivariate Behavioral Research, 28(3), 263-311. https:// doi.org/10.1207/s15327906mbr2803_1

Widaman, K. F. (2007). Common factors versus components: Principals and principles, errors and misconceptions. In R. Cudeck $\&$ R. C. MacCallum (Eds.), Factor analysis at 100: Historical developments and future directions (pp. 177-203). Mahwah, NJ: Lawrence Erlbaum Associates.

Yanyun, Y., \& Green, S. B. (2011). Coefficient alpha: A reliability coefficient for the 21st century? Journal of Psychoeducational Assessment, 29(4), 377-392. https:// doi.org/10.1177/0734282911406668

Young, K. (1996a). Internet addiction: The emergence of a new clinical disorder. CyberPsychology and Behavior, 1(3), 237-244. https://doi.org/10.1089/ cpb.1998.1.237

Young, K. (1996b). Psychology of computer use: XL. Addictive use of the Internet: a case that breaks the stereotype. Psychological Reports, 79(3), 899-902. https://doi.org/10.2466/pr0.1996.79.3.899

Zinbarg, R. E., Yovel, I., Revelle, W., \& McDonald, R. P. (2006). Estimating generalizability to a latent variable common to all of a scale's indicators: A comparison of estimators for $\omega_{h}$. Applied Psychological Measurement, 30(2), 121-144. https:// doi.org/10.1177/0146621605278814

\section{Notas}

* Artículo de Investigación. 